\title{
Generalized bullous morphea
}

\section{Sara Mai', Siham Mansouri', Kaoutar Znati², Meriem Meziane'1, Nadia Ismaili', Laila Benzekri', Karima Senouci' ${ }^{1}$}

\author{
${ }^{1}$ Department of Dermatology, University Hospital Ibn Sina, Rabat, Morocco, ${ }^{2}$ Department of Histopathology, Ibn Sina \\ University Hospital, Rabat, Morocco
}

Corresponding author: Dr. Sara Mai, E-mail: sara250190@gmail.com

\begin{abstract}
Bullous morphea is a rare variant of localized scleroderma characterized by blister formation on sclerous plaques. The pathogenesis of this bullae formation is widely debated. The role of lymphatic obstruction with the sclerous process remains the most plausible hypothesis. We report a case of very extensive generalized morphea complicated with blister formation. The main differential diagnosis for bullous morphea is lichen sclerosus that can also result in bullae in sclerous plaques. The diagnosis of bullous morphea was confirmed by histopathological examination showing typical aspects of scleroderma with a subepidermal blister. The patient was successfully treated with association of Methotrexate, corticosteroids and local care of bullous lesions.
\end{abstract}

Key words: Scleroderma; Bullous morphea; Generalized morphea; Treatment

\section{INTRODUCTION}

Bullous morphea is a rare variant of localized scleroderma characterized by blister formation on sclerous plaques. The pathogenesis of this bullae formation is widely debated. The role of lymphatic obstruction with the sclerous process remains the most plausible hypothesis. We report a case of very extensive generalized morphea complicated with blister formation. The main differential diagnosis for bullous morphea is lichen sclerosus that can also result in bullae in sclerous plaques. The diagnosis of bullous morphea was confirmed by histopathological examination showing typical aspects of scleroderma with a subepidermal blister. The patient was successfully treated with association of Methotrexate, corticosteroids and local care of bullous lesions.

\section{CASE REPORT}

A 66 years old male patient presented with 5 months history of rapidly extensive skin sclerosis affecting the trunk and limbs and sparing the extremities (head, face, hands and feet). The patient had no Raynaud's phenomenon and no systemic symptoms. Physical examination revealed generalized cutaneous sclerosis with a very thick and hard skin covering the trunk and the limbs sparing the folds with severe limitation of motion making it impossible for the patient to stand straight (Fig. 1). There were no signs of sclerodactyly; hands and feet were not affected, nether was the face (Fig. 2). Few days after his admission, patient presented multiple tense bullas in the upper trunk and on the flanks Nikolsky's sign was negative (Fig. 3).

A first skin biopsy had been performed on a sclerous lesion revealing typical histological finding of scleroderma or morphea with epidermis atrophy and hyalinization of the collagen, entrapment of adnexae, and thickening of the collagen bundles in the dermis (Fig. 4).

A second biopsy was performed on a bullous lesion showing a bulla in the papillary dermis with serohematic content (Fig. 5). Direct immunofluorescence was negative. All biological tests, involving anti-DNA and anti-nuclear antibodies (anti-SSA, SSB, Sm/RNP, Scl70) were normal. Borrelia antibody tests were negative.

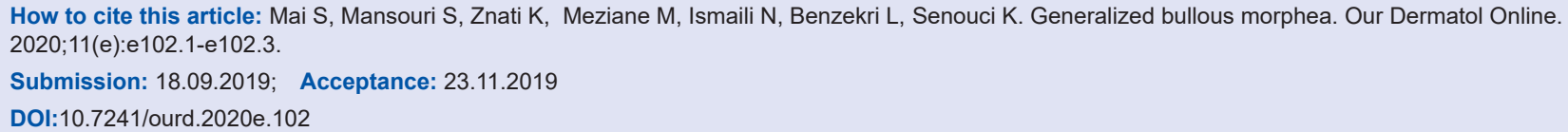




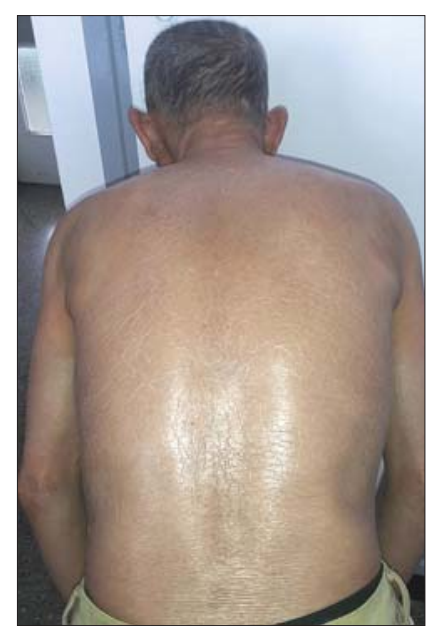

Figure 1: Severe skin sclerosis enclosing the trunk with incapacity to stand straight.

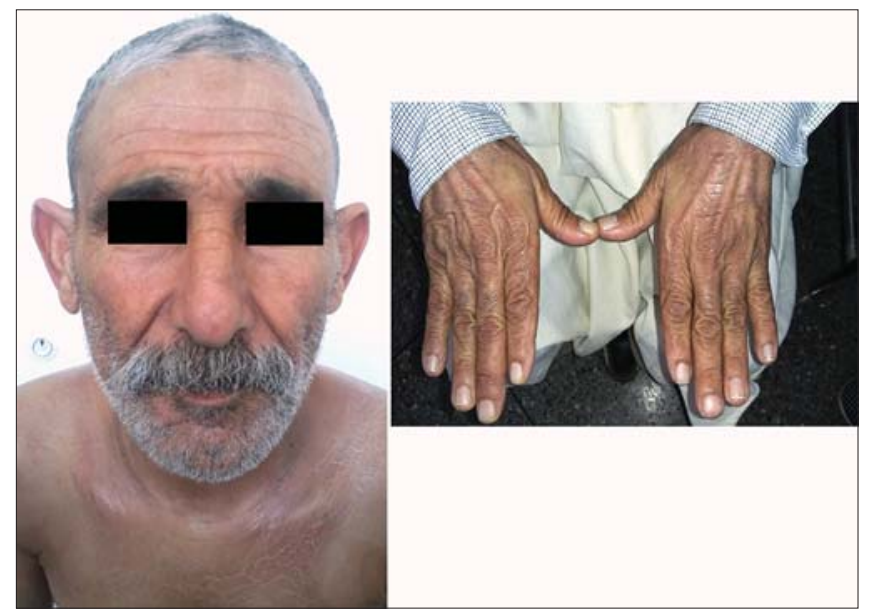

Figure 2: Normal aspect of the skin of the hands and face.

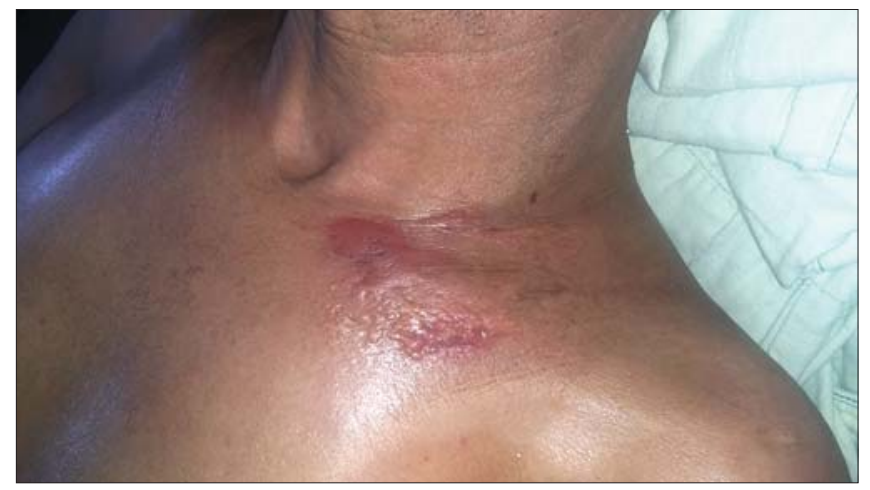

Figure 3: Tense bullae on the upper trunk.

UVAl phototherapy was discussed as first line treatment but since the patient lived in a small town where phototherapy was not available, we had to choose another treatment option and the patient was treated with a combination of oral corticosteroids (prednisone $\mathrm{lmg} / \mathrm{kg} /$ day $)$ and Methotrexate $(15 \mathrm{mg} /$ week) and local care for the bullous lesions.

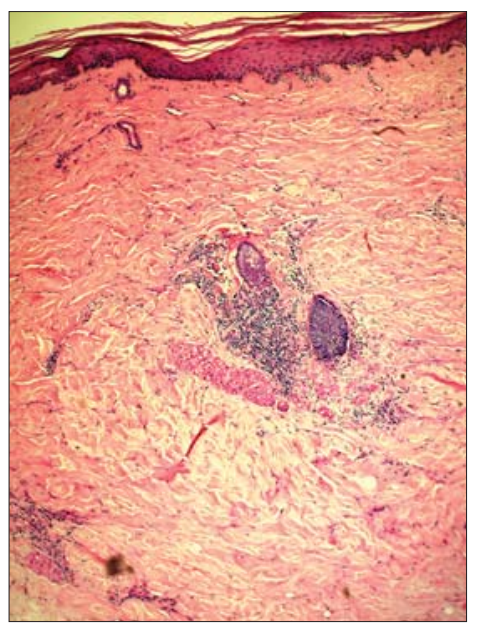

Figure 4: Histological examination (HE stain $\mathrm{x} 20$ ) revealing typical signs of morphea with hyalinization of the collagen, entrapment of adnexae, and thickening of the collagen bundles.

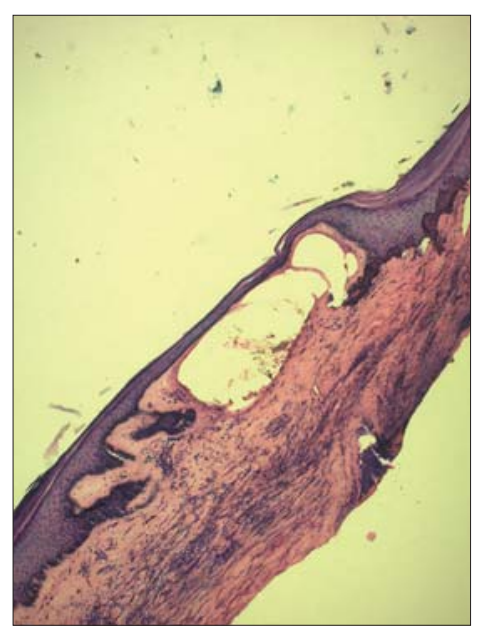

Figure 5: Histological examination (HE stain x20) showing a bulla in the papillary dermis with serohematic content.

After 3 months of treatment the blisters had completely healed with a great improvement in skin sclerosis and its functional consequences. After remission, doses of corticosteroids were tapered progressively. Methotrexate was continued for over a year. No realapse has been noted after 1 year of follow up.

\section{DISCUSSION}

Bullous morphea is a rare entity described for the first time in 1896 by Morraw [1]. Since then, It has rarely been described in the literature. Its frequency is difficult to define since very few series have been reported so far with various rates among cases of scleroderma and morphea. It has been estimated to about 7,5\% of all scleroderma cases [2]. A recent retrospective monocentric study found a rate of $1,4 \%$ of morphea cases in an 11 years period [3]. The exact incidence is unknown. 
Lichen sclerosus (LS) can easily be mistaken with morphea. The presence of bullae is more frequent in LS but histopathological examination can perfectly distinguish the two entities.

The pathogenesis of bullae formation is probably multifactorial and still incompletely understood. The most common theory is that bullae formation is due to lymphatic obstruction by the sclerodermatous process with dilatation of lymphatic vessels. The predilection of bullous lesions in the lower extremities supports this hypothesis. However, although it was described in many cases, this lymphatic obstruction with lymphangiectases is not constant and is also seen in cases of scleroderma without bullous formation. The presence of hemorrhagic content suggests local trauma as a potential mechanism involved in the bullae formation though it can't be considered as the only causative factor. Many other factors have been proposed such as an immune mediated aggression of the basal junction as it is seen in graft vs. host disease. In other reports, authors suggest that perivascular inflammation and especially eosinophils are crucial factors in bullae formation. Rare cases of association of morphea with bullous autoimmune diseases such as pemphigus or epidermolysis bullosa have been reported [3-5].

Treatment of bullous morphea is not consensual and usually depends on the experience on each center. Many treaments have been used such as corticosteroids, colchicine, retinoids, salazopyrin, antimalarials, hydroxychloroquine, pentoxifylline, Broad-band UVA and UVAl phototherapy, and immunomodulatory and immunosuppressive drugs with various results $[2,3]$.

Our patient was treated with association of corticosteroids and Methotrexate because of the severity of his generalized morphea and its extremely fast evolution with serious motion limitation. The treatment allowed complete healing of bullous lesions and progressive improvement of the skin sclerosis and its functional consequences within less than 3 months.

\section{CONCLUSION}

Bullous morphea is a rare variant of localized scleroderma. Pathogenesis of bullae formation in morphea is still incompletely understood but is probably multifactorial. Treatment of bullous morphea remains a challenge because of the lack of randomized controlled trials due to the extreme rarity of this exceptional entity.

\section{Consent}

The examination of the patient was conducted according to the Declaration of Helsinki principles.

\section{REFERENCES}

1. Rowell MR. Lupus erythematosus, scleroderma and dermatomyositis. In: Book A, Wilkinson DS, Ebling FJG, eds.Textbook of Dermatology. Oxford: Blackwell Scientific Publications, 1979: $1198 Đ 1228$.

2. Wagner G, Meyer V, Sachse MM. [Generalized circumscribed scleroderma with blisters]. Hautarzt. 2017;68:566-70.

3. Venturi M, Pinna AL, Pilloni L, Atzori L, Ferreli C, Rongioletti F. Bullous morphoea: a retrospective study. Clin Exp Dermatol. 2017;42:532-5.

4. Fernandez-Flores A, Gatica-Torres M, Tinoco-Fragoso F, García-Hidalgo L, Monroy E, Saeb-Lima M. Three cases of bullous morphea: histopathologic findings with implications regarding pathogenesis. J Cutan Pathol. 2015;42:144-9.

5. Cuellar-Barboza A, Alberto Cardenas-De La Garza J, Daniel Villarreal-Villarreal C, Ocampo-Candiani J, Gomez-Flores M, Welsh $\mathrm{O}$, et al. A Case of bullous morphea resistant to methotrexate and phototherapy successfully treated with mycophenolate mofetil. J Drugs Dermatol. 2018;17:1123-5.

Copyright by Sara Mai, et al. This is an open access article distributed under the terms of the Creative Commons Attribution License, which permits unrestricted use, distribution, and reproduction in any medium, provided the original author and source are credited.

Source of Support: Nil, Conflict of Interest: None declared. 